\title{
HBV vaccination and PMTCT as elimination tools in the presence of HIV: insights from a clinical cohort and dynamic model
}

Anna L. McNaughton ${ }^{1 \dagger}$, José Lourenço ${ }^{2+}$, Louise Hattingh ${ }^{3+}$, Emily Adland ${ }^{4}$, Samantha Daniels ${ }^{3}$, Anriette Van Zyl ${ }^{3}$, Connie S. Akiror ${ }^{5}$, Susan Wareing ${ }^{6}$, Katie Jeffery ${ }^{6}$, M. Azim Ansari ${ }^{1}$, Paul Klenerman ${ }^{1,6}$, Philip J. R. Goulder ${ }^{4}$, Sunetra Gupta ${ }^{2}$, Pieter Jooste ${ }^{3}$ and Philippa C. Matthews ${ }^{1,6^{*}}$ (D)

\begin{abstract}
Background: Sustainable Development Goals set a challenge for the elimination of hepatitis B virus (HBV) infection as a public health concern by the year 2030. Deployment of a robust prophylactic vaccine and enhanced interventions for prevention of mother to child transmission (PMTCT) are cornerstones of elimination strategy. However, in light of the estimated global burden of 290 million cases, enhanced efforts are required to underpin optimisation of public health strategy. Robust analysis of population epidemiology is particularly crucial for populations in Africa made vulnerable by HIV co-infection, poverty, stigma and poor access to prevention, diagnosis and treatment.

Methods: We here set out to evaluate the current and future role of HBV vaccination and PMTCT as tools for elimination. We first investigated the current impact of paediatric vaccination in a cohort of children with and without HIV infection in Kimberley, South Africa. Second, we used these data to inform a new parsimonious model to simulate the ongoing impact of preventive interventions. By applying these two approaches in parallel, we are able to determine both the current impact of interventions, and the future projected outcome of ongoing preventive strategies over time.

Results: Existing efforts have been successful in reducing paediatric prevalence of HBV infection in this setting to $<1 \%$, demonstrating the success of the existing vaccine campaign. Our model predicts that, if consistently deployed, combination efforts of vaccination and PMTCT can significantly reduce population prevalence (HBsAg) by 2030, such that a major public health impact is possible even without achieving elimination. However, the prevalence of HBV e-antigen (HBeAg)-positive carriers will decline more slowly, representing a persistent population reservoir. We show that HIV co-infection significantly reduces titres of vaccine-mediated antibody, but has a relatively minor role in influencing the projected time to elimination. Our model can also be applied to other settings in order to predict impact and time to elimination based on specific interventions.

(Continued on next page)
\end{abstract}

\footnotetext{
* Correspondence: philippa.matthews@ndm.ox.ac.uk

${ }^{\dagger}$ Anna L. McNaughton, José Lourenço and Louise Hattingh contributed equally to this work.

${ }^{1}$ Nuffield Department of Medicine, Peter Medawar Building for Pathogen Research, South Parks Road, Oxford OX1 3SY, UK

${ }^{6}$ Department of Infectious Diseases and Microbiology, Oxford University Hospitals NHS Foundation Trust, John Radcliffe Hospital, Headley Way, Oxford OX3 9DU, UK

Full list of author information is available at the end of the article
}

(c) The Author(s). 2019 Open Access This article is distributed under the terms of the Creative Commons Attribution 4.0 International License (http://creativecommons.org/licenses/by/4.0/), which permits unrestricted use, distribution, and reproduction in any medium, provided you give appropriate credit to the original author(s) and the source, provide a link to the Creative Commons license, and indicate if changes were made. The Creative Commons Public Domain Dedication waiver (http://creativecommons.org/publicdomain/zero/1.0/) applies to the data made available in this article, unless otherwise stated. 
(Continued from previous page)

Conclusions: Through extensive deployment of preventive strategies for HBV, significant positive public health impact is possible, although time to HBV elimination as a public health concern is likely to be substantially longer than that proposed by current goals.

Keywords: Hepatitis B virus, Epidemiology, Africa, Antibodies, Immunisation, PMTCT, Sustainable Development Goals, HIV, Vaccination, Elimination

\section{Background}

The vaccine against hepatitis $B$ virus (HBV) infection is one of the cornerstone strategies underpinning progress towards Sustainable Development Goals (SDGs) and World Health Organization (WHO) targets for HBV elimination as a public health threat by the year $2030[1,2]$. The global health community, including organisations such as WHO and the Centre for Disease Control, classifies HBV prevalence into high, intermediate or low, using widely cited thresholds of $\geq 8 \%, 2-7 \%$ and $<2 \%$, respectively [3, 4]. Many studies from Africa report a high HBV prevalence, and reports exceeding $15 \%$ are not uncommon $[3,5]$. However, there is considerable heterogeneity within the continent [5]. Populations in southern Africa are particularly vulnerable to HBV-related morbidity and mortality due to the high prevalence of infection in many regions $[3,5,6]$, co-endemic HIV infection [7], poor access to screening and diagnostics, limited access to antiviral therapy, stigma, and chronic neglect of education, research and resources $[8,9]$. In this region, a substantial burden of HBV transmission occurs early in life, either vertically from mother to child or through horizontal acquisition in young children [10]. The HBV vaccine has been progressively rolled out as part of the WHO Expanded Programme on Immunisation (EPI) over the past two decades [10], but the first vaccine dose is often postponed until age 6 weeks, when it is given together with other routine immunisations [11].

Vaccine deployment can be difficult to measure, as many children in Africa are born outside healthcare settings, there are no robust data regarding coverage of the three dose regimen [10], and different immunological correlates of protection have been applied [12, 13]. To accelerate progress towards elimination goals, suggested modifications to vaccine schedules have included shifting the first dose to be given at birth [14], additional doses in the context of HIV infection [15, 16], booster doses in individuals whose antibody titre fails to meet a target threshold [12], and catch-up vaccination campaigns for adolescents and adults. Prevention of mother to child transmission (PMTCT) can be achieved through a combination of accelerated vaccination (starting with a birth dose), maternal antivirals in pregnancy, and use of hepatitis B immunoglobulin (HBIg) prophylaxis [17]. However, antenatal HBV screening is still not routinely undertaken in many settings in Africa, and interventions are thus not offered to women at highest risk of transmitting HBV infection to their babies.

There is a lack of robust data to inform which of these measures, individually or in combination, is most effective. Given the resource limitations of many settings in which HBV represents a public health challenge, there is an urgent need to underpin interventions with an evidence base derived both from careful observation of the existing impact of vaccination and PMTCT, and from projections regarding ongoing impact.

On these grounds, we have set out to collect a detailed dataset to provide a snapshot of a population in South Africa in which HBV and HIV infections are co-endemic, first seeking evidence of the impact of the current immunisation schedule in children and then assessing the extent to which interventions could be predicted to achieve elimination targets. We built on this framework by adding data assimilated from the wider published literature to model the effects of different HBV vaccine deployment strategies, either alone or in combination with enhanced PMTCT measures.

To date, few attempts have been made to model the impact of HBV vaccination, with one study modelling the global prevalence of current intervention efforts [18], and another that scrutinises the combined impact of broad HBV elimination strategies [19]. In this instance, we report a novel approach founded on primary clinical data, quantifying the individual and combined impact of childhood vaccination and PMTCT, and addressing the specific impact of co-endemic HIV infection. Combining output from a clinical dataset together with a dynamic model provides a synergistic approach to characterising the problem and projecting the effects of vaccination. Taking the evidence together, we conclude that while vaccination is a fundamental part of global elimination strategy and is highly effective in preventing infection in individual children, there remains an urgent need for rigorous, enhanced deployment of parallel strategies including education, diagnostics, antiviral therapy, and the ongoing quest for a cure.

\section{Methods}

\section{Ethics approval}

Ethics approval was obtained from the Ethics Committee of the Faculty of Health Science, University of the Free State, Bloemfontein, South Africa (HIV Study Ref: ETOVS 
Nr 08/09 and COSAC Study Ref: ECUFS NR 80/2014), and from the Oxfordshire Research Ethics Committee A, ref 06/Q1604/12. Written consent for enrollment into the study was obtained from the child's parent/guardian.

\section{Study cohorts}

Recruitment was undertaken in Kimberley, South Africa. In this setting, a standard three-dose HBV immunisation schedule is deployed in infants, with the first dose at 6 weeks. A previous study of HBV serology in adults in the same setting found HBsAg prevalence of 9.5\% (55/579) [6]. Children were recruited as part of the Co-infection in South-African Children ('COSAC') study as previously described $[20,21]$. The lower age limit of recruitment was 6 months in order to limit the detection of maternal anti-HBs.

Children were recruited as follows:

1. HIV-negative children age $6-60$ months $(n=174)$, recruited through the Kimberley Respiratory Cohort (KReC) as previously described [20]. These children were admitted to hospital between July 2014 and August 2016 with a clinical diagnosis of respiratory tract infection. $\mathrm{KReC}$ children were confirmed HIV-negative in 163 cases (93.7\%). A further 11 children did not have an HIV test result recorded, but were assumed to be HIV-negative based on the clinical data recorded at the time of admission to hospital.

2. HIV-positive children were recruited primarily from HIV out-patient clinics between September 2009 and July 2016 as previously described [20, 21]. We recorded date of commencement of anti-retroviral therapy (ART), CD4+ T cell count and percentage, and HIV RNA viral load using the time point closest to the sample that was analysed for HBV serology. For the purpose of analysis, we divided these into two groups according to age:

i. Age 6-60 months; $n=136$. This group was selected to match the age range of the HIV-negative group and also included five children who were initially screened for the $\mathrm{KReC}$ cohort but tested HIV-positive.

ii. Age $>60$ months (range 64-193 months); $n=92$.

At the time of undertaking this study, children were immunised with three doses of a monovalent $\mathrm{HBV}$ vaccine (Biovac Paed). Where possible, we recorded the number of HBV vaccine doses received based on the Road to Health Book (RTHB). The characteristics of the cohorts are summarised in Table 1 and all metadata can be found in Additional file 1.

\section{Laboratory assessment of HBV status}

Testing for hepatitis B serum markers and DNA was performed as previously described, and in keeping with recent implementation of HBV screening in Kimberley [21]. Briefly, HBsAg testing was carried out in Kimberley Hospital, South Africa, using the magnetic parcel chemiluminometric immunoassay (MPCI; Advia Centaur platform). Confirmatory HBsAg testing was carried out by the clinical microbiology laboratory at Oxford University Hospitals (OUH) NHS Foundation Trust, Oxford, UK (Architect i2000). For all samples, anti-HBs and anti-HBc testing were carried out by the OUH laboratory (Architect i2000). Limit of detection of the anti-HBs assay was $10 \mathrm{mIU} / \mathrm{ml}$.

\section{Threshold for vaccine-mediated immunity}

Studies variably quote anti-HBs titres of $\geq 10 \mathrm{mIU} / \mathrm{ml}$ or $\geq 100 \mathrm{mIU} / \mathrm{ml}$ as a correlate of protection; UK recommendations for testing HBV immunity advocate the more stringent criterion of an anti-HBs titre of $\geq 100$ $\mathrm{mIU} / \mathrm{ml}$ [12], while early vaccine studies suggest a titre of $\geq 10 \mathrm{mIU} / \mathrm{ml}$ as a clinically relevant threshold for protection $[13,22]$. We have presented our results pertaining to both thresholds.

\section{Statistical analysis}

Data from the cohort was analysed using GraphPad Prism v.7.0. We determined significant differences between sub-sets within the cohort using Mann-Whitney $U$ tests for non-parametric data, Fisher's exact test for categorical variables and Spearman's correlation coefficient for correlation between data points.

Table 1 Characteristics of three paediatric study cohorts, comprising 402 children, recruited from Kimberley Hospital, South Africa

\begin{tabular}{llll}
\hline Cohort & HIV negative; KReC (age $\leq 60$ months) & HIV positive cohort (age $\leq 60$ months) & HIV positive cohort (age $>60$ months) \\
\hline Number of subjects & 174 & 136 & 92 \\
Age range in months & $8-58$ & $6-60$ & $64-193$ \\
Median age in months (IQR) & $18(12-26)$ & $29(18-40)$ & $137(122-154)$ \\
Sex (\% male) & 55.4 & 44.9 & 45.6 \\
\hline
\end{tabular}

KReC Kimberley Respiratory Cohort, IQR interquartile range 
Mathematical model of HBV transmission and prevention Here, we summarise the modelling framework, but include a detailed description of the ODE system, model parameters, and Bayesian data fitting approach in Additional file 2. We developed a dynamic model based on ordinary differential equations (ODE), for which parameterisation of HBV transmission and prevention was based both on our Kimberley paediatric cohort and current literature estimates.

In summary, the model takes into consideration the proportion of the population susceptible to HBV infection $(\mathrm{S})$, those with chronic infection $(\mathrm{C})$ and acute infection (I), those who are immune as a result of recovery from prior infection $(\mathrm{R})$ and those who are immune as a result of vaccination (V) (Fig. 1). For simplicity, and assuming vaccination takes place early in life, all individuals are assumed to be born either susceptible (Z) or vaccinated (Z'). Chronic carriers $(\mathrm{C})$ are divided into $\mathrm{HBeAg}$-positive $(\mathrm{C}+)$ and $\mathrm{HBeAg-negative}(\mathrm{C}-)$ to further allow for different parameterisation (e.g. transmission potential) between these two epidemiologically distinct states. To be able to parameterise epidemiological traits by age, (e.g. probability of chronicity, or decay of vaccine-induced protection) susceptible (S) and vaccinated (V) individuals are divided into three subgroups representing infants (i, < 1 years of age), children (c, 1-6 years of age) and older individuals (whom we abbreviate to 'adults', a, but comprise children age $>6$ years, adolescents and adults). The probability of developing chronicity decreases with age, with $(1-\psi)$ for infants, $(1-\varepsilon)$ for children and $(1-\gamma)$ for older individuals. Vertical transmission takes place from mothers with chronic infection and is dependent on their HBeAg serostatus (not shown on diagram). HBeAg-positive chronic carriers $(\mathrm{C}+)$ may become $\mathrm{HBeAg-negative} \mathrm{at} \mathrm{a} \mathrm{rate} \theta$. HBeAg-negative chronic carriers $(\mathrm{C}-)$ can clear infection spontaneously at a rate $\rho$, entering the anti-HBc-positive, $\mathrm{HBsAg}$-negative state (R). Acute infections (I) are cleared at a rate $\sigma$, also entering the recovered class $(R)$.

Vaccinated individuals ( $\mathrm{Vi}, \mathrm{Vc}, \mathrm{Va}$ ) are under the same HBV acute and chronic infection rules as susceptible individuals ( $\mathrm{Si}, \mathrm{Sc}, \mathrm{Sa})$, but are further assumed to have vaccine-induced age-dependent protection against infection $(\Delta \mathrm{i}, \Delta \mathrm{c}, \Delta \mathrm{a})$. For simplicity, we assume that vaccine-induced protection was equivalent to reducing susceptibility to infection potential $(\lambda)$, e.g. $\Delta i=1$ would be $100 \%$ reduction in susceptibility, or $100 \%$ vaccine efficacy against infection. Interventions include routine vaccination at birth (affecting Z') and other ages (affecting $\omega \mathrm{a}, \omega \mathrm{c}$ ), as well as PMTCT at birth (affecting Z, Z') and catch-up events (not shown in diagram).

We used a Bayesian Markov-chain Monte Carlo (bMCMC) approach to fit the dynamic model to the local demographic and epidemiological setting of Kimberley before projecting the impact of interventions (Additional file 2: Figure S1). The bMCMC used informative priors for ODE model parameters for which robust literature support exists. Two parameters $(\rho, \theta)$ were left with uninformed priors (uniform, from 0 to 1 ), for which we later checked if the fitted bMCMC solution recovered posteriors of these parameters compatible with

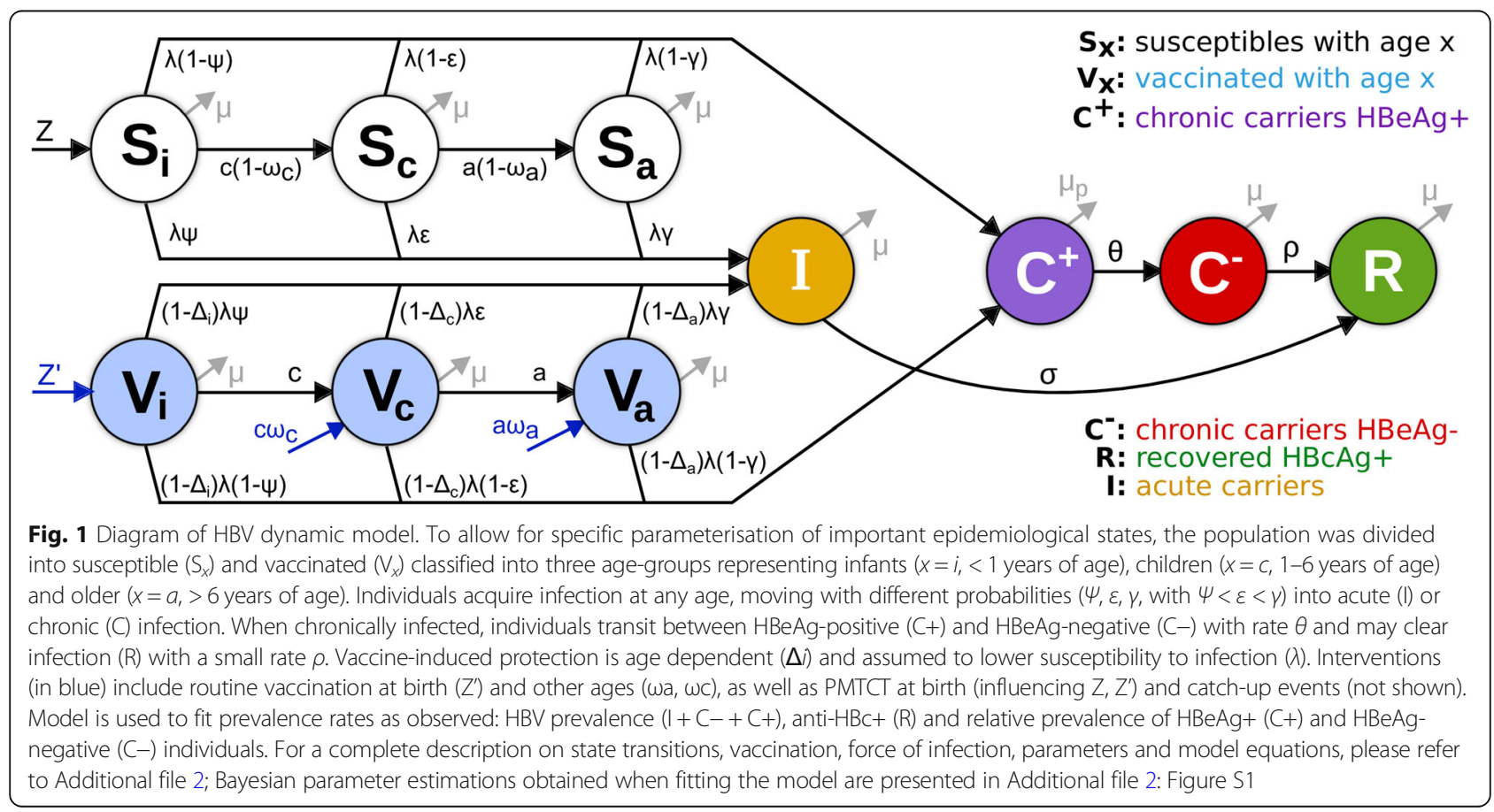


current literature knowledge (as partial validation of the fitted solution). Informed by the clinical cohort data described above, natural decay (age-effects) and the effects of HIV sero-status on vaccine-induced protection $(\Delta \mathrm{i}, \Delta \mathrm{c}$, $\Delta \mathrm{a}$ ) are taken into account (Additional file 2: Figure S2).

\section{Measuring impact of interventions}

SDGs for the year 2030 have been set out in the WHO Global Health Sector Strategy on Viral Hepatitis (GHSSVH) [2]. Given the public health relevance of chronic infections, in particular of HBeAg-positive infections, we measured impact of interventions based on two targets:

i. The WHO target for a $90 \%$ reduction in $\mathrm{HBsAg}$ incidence, based on the assumption that this applies to chronic infection (WHO goals also refer to HBsAg prevalence, for which modelling data are presented in Additional file 2).

ii. An additional target for reduction of $\mathrm{HBeAg}$-positive prevalence to 1 in $1000(0.1 \%)$ in the whole population.

\section{Results}

\section{Serological evidence of exposure to HBV infection}

From our cohort of 402 children in Kimberley, South Africa, three were HBsAg-positive (0.7\%; Table 2), indicating active infection. This HBsAg prevalence is significantly lower than in adults in a comparable study population (e.g. $11.1 \%$ in a previous study [6]; $p<0.0001$ ), but is typical of a population with widespread vaccine coverage [10], highlighting the positive impact of the EPI programme to date. Exposure to HBV infection was measured using anti-HBc antibody; this was detected in three children (0.7\%), one of whom was also HBsAg-positive. The other two were HBsAg-negative, indicating previous HBV exposure and clearance.

\section{Evidence of vaccination and immunity to $\mathrm{HBV}$ in children aged $\leq 60$ months}

We collected written evidence of immunisation from the RTHB in $90.8 \%$ HIV-negative (KReC) subjects and $6.3 \%$ of HIV-positive subjects (total $41.3 \%$ of the entire study cohort). None of the HBsAg-positive children attended with a written vaccination record. Among those with a RTHB record, $81.3 \%$ of HIV-negative and $100 \%$ of HIV-positive children were recorded as having received three HBV vaccine doses. Among all children age $\leq 60$ months, 238/310 (77\%) had an anti-HBs titre $\geq 10 \mathrm{mIU} / \mathrm{ml}$ suggesting some degree of vaccine-mediated immunity (Fig. 2A). The median anti-HBs titre in HIV-negative children was significantly higher than among the HIV-positive group $(196 \mathrm{mIU} / \mathrm{ml}$, vs. $11 \mathrm{mIU} / \mathrm{ml}$, respectively, $p<0.0001)$ (Fig. 2B). There was no detectable anti-HBs antibody in $3.4 \%$ of HIV-negative vs. $47.8 \%$ of HIV-positive children $(p<0.0001)$. Irrespective of the antibody titre used as a threshold for immunity, anti-HBs was higher in HIV-negative compared to HIV-positive children (Fig. 2C). There was no significant difference in anti-HBs titres between male and female participants, either with or without HIV infection ( $p=0.49$ and 0.31 respectively, data not shown).

Table 2 Detailed information and serological profiles of five children from Kimberley, South Africa, with serological evidence of current or previous infection with HBV (based on positive $\operatorname{HBsAg}(n=3)$ and/or anti-HBC $(n=3)$ )

\begin{tabular}{|c|c|c|c|c|c|}
\hline Subject ID & K306 & K405 & KReC51 & KReC151 & K093 \\
\hline Cohort & $\mathrm{HIV}+$ age $\leq 60$ months & $\mathrm{HIV}+$ age $\leq 60$ months & $\mathrm{KReC}$ & KReC & $\mathrm{HIV}+$ age $>60$ months \\
\hline Sex & $\mathrm{F}$ & $\mathrm{F}$ & $\mathrm{F}$ & M & $\mathrm{F}$ \\
\hline $\begin{array}{l}\text { Age (months) at } \\
\text { time of sampling }\end{array}$ & 18 & 37 & 20 & 15 & 118 \\
\hline HIV infection & Positive & Positive & Negative & Negative & Positive \\
\hline ART $^{a}$ (if HIV positive) & Yes & Yes & $\mathrm{n} / \mathrm{a}$ & $\mathrm{n} / \mathrm{a}$ & No \\
\hline $\begin{array}{l}\text { Number of doses } \\
\text { of HBV vaccine }\end{array}$ & NK & NK & NK & 3 & NK \\
\hline HBsAg result ${ }^{b}$ & Detected & Detected & Detected & Not detected & Not detected \\
\hline Anti-HBC result ${ }^{c}$ & Not detected & Not detected & Detected & Detected & Detected \\
\hline HBeAg result ${ }^{d}$ & Not done & Not done & Detected & Not done & Not done \\
\hline Anti-HBs result ${ }^{\mathrm{e}}$ & Not detected & Not detected & Not detected & Detected & Not detected \\
\hline Interpretation & Active infection & Active infection & Active infection & Immunised, infected and cleared & Infected and cleared \\
\hline
\end{tabular}

${ }^{\mathrm{a} A R T}$ indicates the participant was receiving anti-retroviral therapy to treat HIV infection; ${ }^{\mathrm{b}}$ hepatitis B surface antigen test; ${ }^{\mathrm{C}}$ hepatitis B core antibody test;

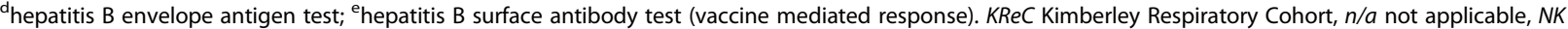
not known 

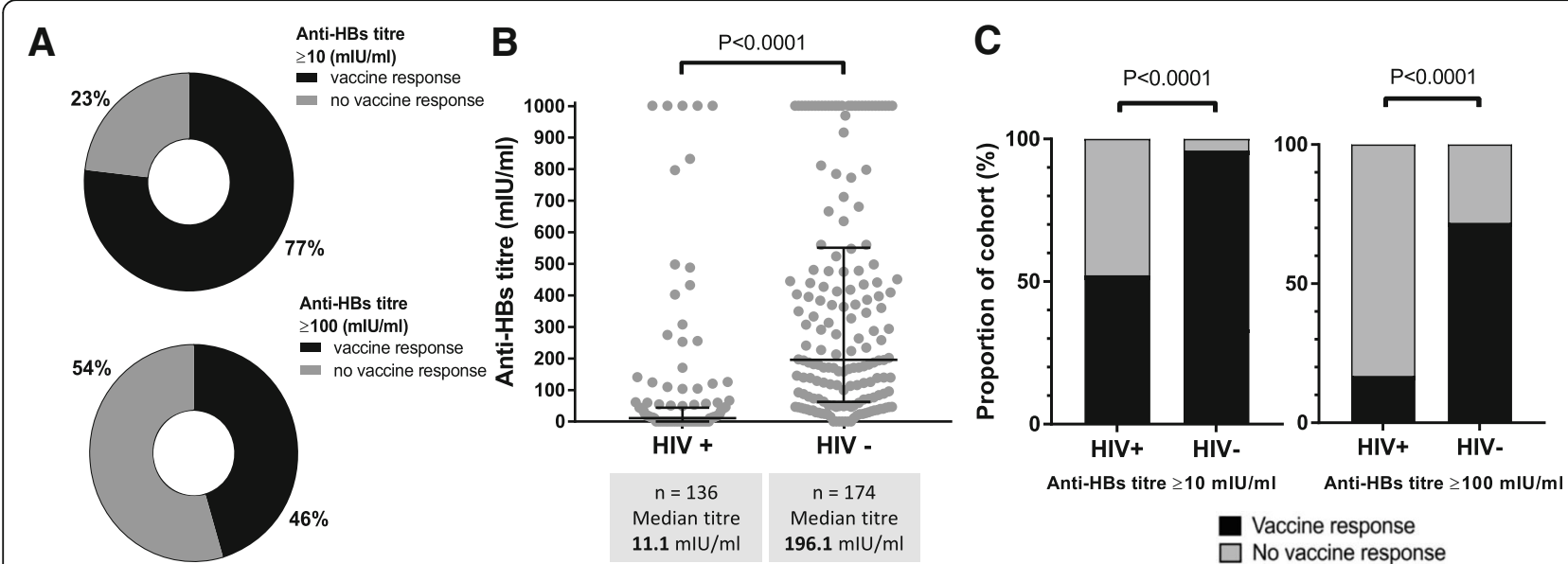

Fig. 2 Hepatitis B surface antibody (anti-HBs) titres mediated by vaccination in the entire cohort and in HIV-positive (HIV+) and HIV-negative (HIV-) children aged 6-60 months in Kimberley, South Africa. A Doughnut charts indicating the proportion of the cohort achieving anti-HBs titres of $\geq 10 \mathrm{mlU} / \mathrm{ml}$ or $\geq 100 \mathrm{mlU} / \mathrm{ml}$. B Scatter plot representing vaccine-mediated antibody titres according to HIV-status, indicating median and interquartile ranges ( $p$ value by Mann Whitney $U$ test). C Proportion of children with anti-HBs $\geq 10 \mathrm{mlU} / \mathrm{ml}$ or $\geq 100 \mathrm{mlU} / \mathrm{ml}$ according to HIV-status ( $p$ values by Fisher's exact test)

\section{Waning of vaccine response with age}

HIV-positive children with anti-HBs titres $\geq 100 \mathrm{mIU} / \mathrm{ml}$ were significantly younger than those with lower antibody titres (median age 17 months vs. 31 months, $p=0.0008$ ), while no such difference was observed within the HIV-negative group (Fig. 3A). Using the lower threshold of $\geq 10 \mathrm{mIU} / \mathrm{ml}$, we found no significant difference by age in either the HIV-positive or the HIV-negative groups ( $p=0.17$ and 4.48 respectively, data not shown). To expand our view of the HIV-positive group, we also added analysis of an older cohort (92 children aged $>60$ months) and demonstrated that anti-HBs titres were significantly lower in this older group $(p<0.0001)$, with only $2 / 92$ subjects (2.2\%) achieving a detectable anti-HBs titre (Fig. 3B). Anti-HBs titres waned significantly with age up to age 60 months in HIV-positive children (Fig. 3C; $p=0.004$ ). We observed a similar trend in the HIV-negative cohort, but this did not reach a statistical significance (Fig. 3C; $p=0.07$ ). The proportion of HIV-positive subjects with a detectable anti-HBs titre declined steadily with age in the cohort, contrasting to the trend in HIV-negative subjects, where individuals maintained protective anti-HBs titres despite a trend towards decreasing mean titres (Fig. 3C).

\section{Stratification of vaccine responses by anti-retroviral therapy (ART) among HIV-positive children}

For HIV-positive children aged $\leq 60$ months, ART treatment data were available for $79 \%$ of subjects. Within this group, $71 \%$ were receiving ART at the time we tested for anti-HBs, and had received a median of 20 months of treatment (IQR 6-33 months). Comparing anti-HBs titres between ART-treated vs. untreated children, we found no significant difference $(p=0.72 ; 76$ ART-treated, median anti-HBs $13.3 \mathrm{mIU} / \mathrm{ml}$ and 31 untreated children, median anti-HBs $14.1 \mathrm{mIU} / \mathrm{ml}$, data not shown). There was also no difference between anti-HBs titres of children treated for $\leq 12$ months vs. $>12$ months $(p=0.50$, data not shown). We did not examine the effect of ART on anti-HBs titres in children $>60$ months due to the low numbers of subjects with a detectable anti-HBs titre $(n=2)$.

\section{Odds of developing an anti-HBs response}

We used an odds ratio (OR) analysis to identify factors associated with vaccine-mediated protection (Fig. 3D). HIV-positive status was associated with lack of protection, for antibody titres of both $<10 \mathrm{mIU} / \mathrm{ml}$ (OR 26.2, 95\% CI 11.2-58.6) and < $100 \mathrm{mIU} / \mathrm{ml}$ (OR 11.6, 95\% CI 6.7-20.4). In contrast, younger age (<24 months) was protective in HIV-positive subjects, (for anti-HBs $<10$ $\mathrm{mIU} / \mathrm{ml}$, OR 0.3 , 95\% CI 0.2-0.5, and for anti-HBs $<100 \mathrm{mIU} / \mathrm{ml}$, OR 0.3, 95\% CI 0.2-0.4). Gender, ART, CD4+ count, CD4+ ratio, and HIV viral load were not found to be significantly predictive of anti-HBs titres at either threshold.

\section{Fitting of the dynamic model to local HBV epidemiology}

The mathematical model was used in three steps, as follows. Step 1: calibration to a demographic background by estimation of rates of movement between the age-structured susceptible classes, with the aim of deriving similar age-proportions to Kimberley (in the absence of transmission). Step 2: calibration to the transmission background without interventions, in which local HBV prevalence (HBsAg, or sum of infected), prevalence of $\mathrm{HBV}$ exposure (recovered 


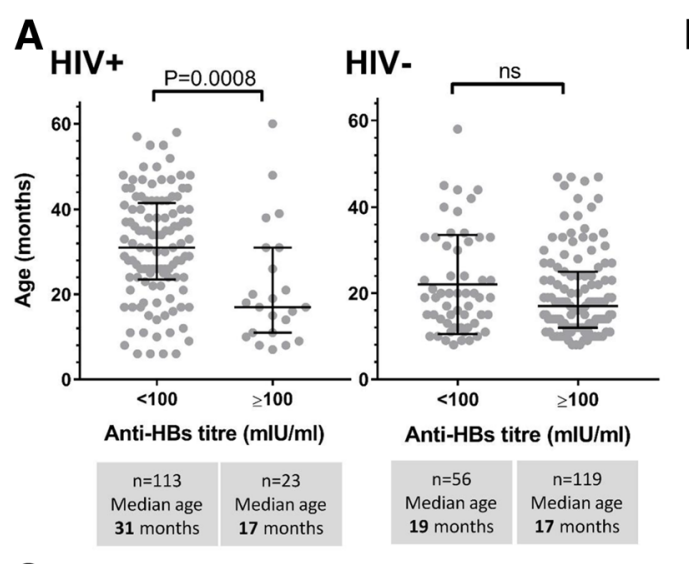

B
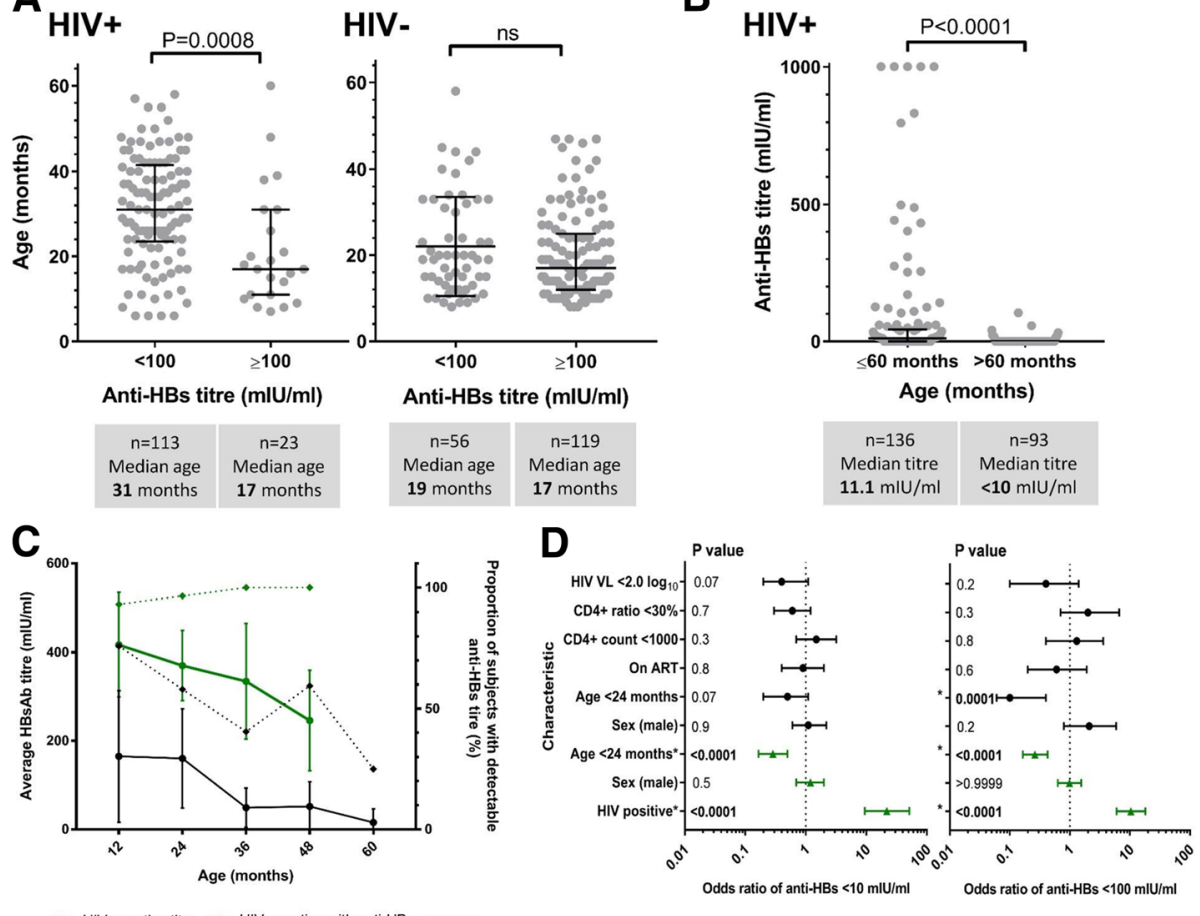

$\rightarrow$ HIV negative titre $\rightarrow$ HIV negative with anti-HBs repsonse

HIV-postive subjects $\leq 60$ months $\rightarrow-$
All subjects $\leq 60$ months -

Fig. 3 Relationship between age and vaccine-mediated hepatitis B surface antibody (anti-HBs) titres in HIV-positive and HIV-negative children in Kimberley, South Africa. A Ages of children attaining anti-HBs titres $\geq 100 \mathrm{mIU} / \mathrm{ml}$ for HIV-positive and HIV-negative children age 6-60 months. Median ages, interquartile ranges, and $p$ values by Mann-Whitney $U$ test are indicated. B Relationship between age and vaccine-mediated Ab titre among HIV-positive children including those age 6-60 months and an older cohort age $>60$ months (range 64-193 months). $p$ value by Mann Whitney $U$ test. C Anti-HBs titre and proportion of subjects with a detectable titre for HIV-positive and HIV-negative children according to age. On the solid lines, each point represents the mean titre (with 95\% confidence intervals) for the group of children aged $\leq 12$ months (1 year), 13-24 months (2 years), 25-36 months (3 years), 37-48 months (4 years), and 49-60 months (5 years). For the same groups of children, the dotted lines represent the proportion of subjects with a detectable titre. Trends within the data were assessed using linear regression analysis. D Odds ratios for protective response to HBV vaccination in children age 6-60 months in Kimberley, South Africa, are shown for anti-HBs titre $<10 \mathrm{mlU} / \mathrm{ml}$ and $<100 \mathrm{mlU} / \mathrm{ml}$ in the whole cohort (green) and in HIV-positive children (black). Statistically significant OR are denoted by asterisk and significant $p$ values are indicated in bold

class $\mathrm{R}$ ) and relative proportion of $\mathrm{HBeAg}$-negative and HBeAg-positive among chronic carriers were fitted. Step 3: the model was run until it reached equilibrium, effectively reproducing the (mean) desired proportions of step 2, at which point interventions were started and the model was tracked for 1000 years. Details on all steps, the methods and parameters involved can be found in Additional file 2.

In step 2, when calibrating to the transmission background, the model closely reproduced the desired variables (fitted distributions and estimated parameter posteriors in Additional file 2: Figure S1 A1-2). For parameters for which insufficient support was found in the literature, the resulting posteriors were well behaved (Additional file 2: Figure S1 B), while for parameters using informative priors the posteriors matched the priors well (Additional file 2: Figure S1 C-D). Overall, the obtained bell-shaped posteriors highlighted a good data fit and no identifiability issues with the bMCMC approach. Based on these results, we next simulated stochastic projections of intervention impact.

\section{Model projection of the impact of routine neonatal vaccination and PMTCT alone}

Based on SDGs [2], Fig. 4 shows the projected impact of varying coverage of neonatal vaccination and PMTCT. Both HBsAg incidence (Fig. 4A1) and HBeAg-positive prevalence (Fig. 4B1) reduce faster with increasing neonatal immunisation coverage, resulting in shorter times to reach SDGs (Fig. 4A2, B2). Importantly, even immunisation of $100 \%$ of neonates is predicted to take $\sim 99$ years (95\% CI 61-186) for the HBsAg incidence target (Fig. 4A2) and $~ 175$ years (95\% CI 103-278) for the HBeAg prevalence target (Fig. 4B2).

With PMTCT interventions, both HBsAg incidence (Fig. 4C1) and $\mathrm{HBeAg}$ prevalence (Fig. 4D1) reduced 


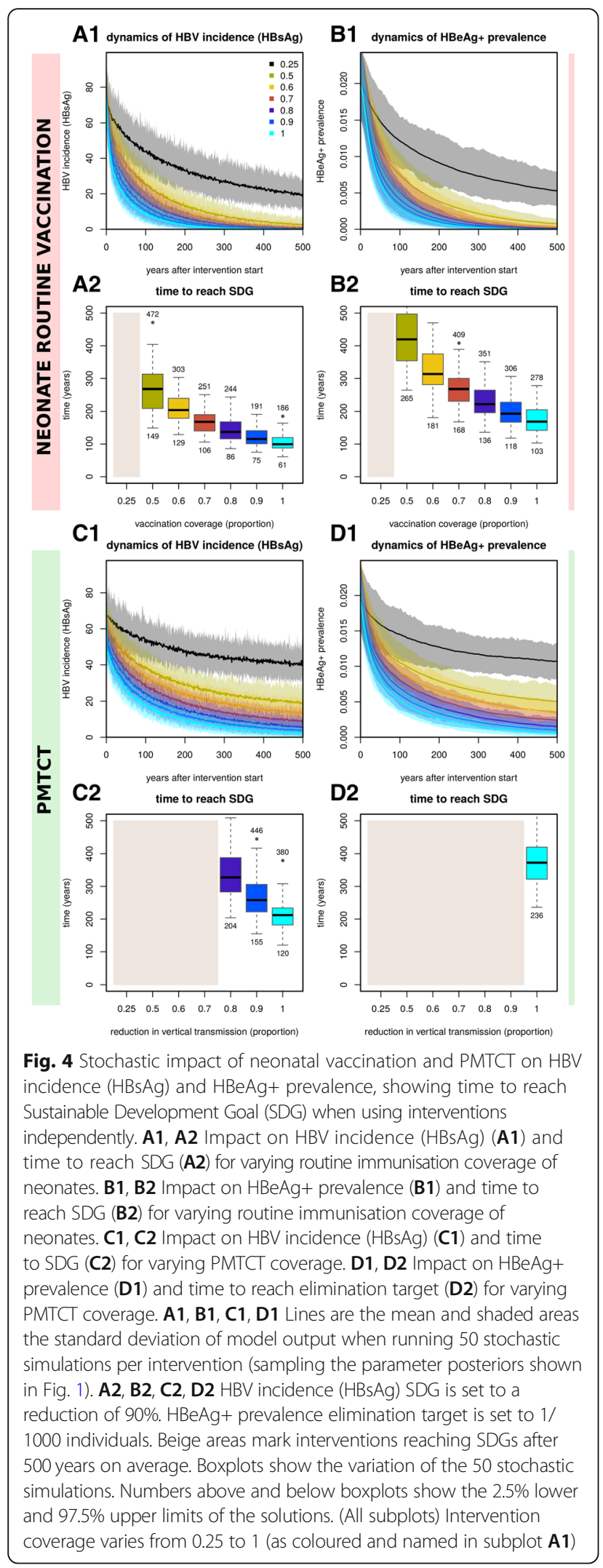

faster in time for increasing efforts, resulting in shorter times to reach the elimination targets (Fig. 4C2, D2). However, the impact of PMTCT was smaller than neonatal vaccination, resulting in longer times to reach SDGs. In fact, for the majority of PMTCT effort levels simulated, SDGs could not be reached within 500 years (beige areas in Fig. 4C2, D2). For HBeAg-positive prevalence, only when PMTCT effort was 1 (i.e. no vertical transmission), was the elimination target attainable within 500 years.

For complementary results using neonatal vaccination and PMTCT with impact on total prevalence (acute and chronic), see Additional file 2: Figure S3, and for time to reach SDG targets, see Additional file 2: Figure S4.

\section{Modelling progress towards HBV elimination by the year 2030 based on combinations of neonatal vaccination and PMTCT}

We projected impact of combined interventions by the year 2030 (Fig. 5A1, B1), and predicted the year at which SDGs would be reached (Fig. 5A2, B2). Strikingly, HBsAg incidence could already have been reduced by $>$ 90\% (Fig. 5A1) if both neonatal vaccination and PMTCT had been deployed at $100 \%$ coverage since they became available in 1995 (mean predicted year of elimination 2017; Fig. 5A2). In reality, complete coverage is not possible, and we therefore projected outcomes based on < $100 \%$ coverage. For example, combining neonatal vaccination and PMTCT with $90 \%$ coverage of each since 1995 would achieve the HBsAg incidence target by 2028 ; if this is reduced to $80 \%$ coverage, then goals would be attained by 2044 . To achieve the target reduction in HBeAg prevalence, modelled on $90 \%$ coverage and $80 \%$ coverage of interventions, the projected years are 2072 and 2096 respectively (Fig. 5B1, B2).

Overall, the highest probability of achieving elimination targets is through a combination of $100 \%$ neonatal vaccination coverage and PMTCT (Fig. 6A1, red line). Based on $90 \%$ coverage of neonatal vaccine and PMTCT (Fig. 6A1, green line) as proposed in WHO's GHSSVH [2], there was only $50 \%$ probability of reaching the HBsAg incidence target by 2030 and approaching $100 \%$ probability only by 2050 . For the target based on HBeAg prevalence, the probabilities of achieving the goal were pushed forward by approximately four decades (Fig. 6A2).

For complementary results on impact and time to reach SDGs when considering combinations of PMTCT and routine vaccination at the age of 6 , see Additional file 2: Figure S5, and for combinations of PMTCT and neonate routine vaccination plus a complete catch-up campaign, see Additional file 2: Figure S6. 

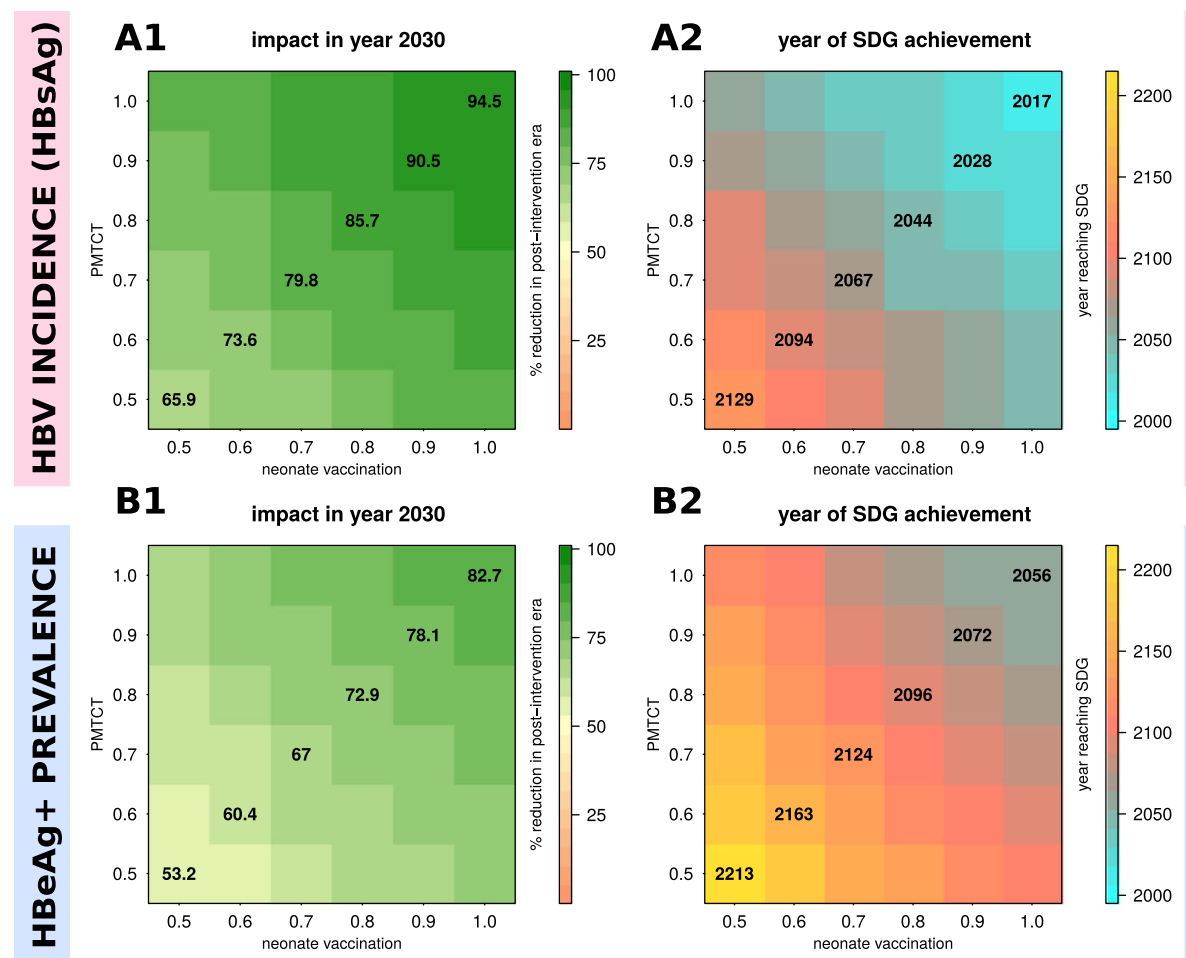

Fig. 5 Sensitivity of mean intervention impact on HBV incidence (HBsAg) and $\mathrm{HBeAg}+$ prevalence based on combinations of routine neonatal vaccination and PMTCT. A1, A2 Mean impact of interventions on HBV incidence (HBsAg) (A1) and mean time to reach Sustainable Development Goals (SDGs) (A2). B1, B2 Mean impact of interventions on HBeAg+ prevalence (B2) and mean time to reach elimination target (B2). For all subplots, impact is shown as percent reduction in incidence or prevalence compared to pre-intervention levels (e.g. 50 indicates a $50 \%$ reduction compared to before the start of the intervention). $\mathrm{HBV}$ incidence (HBsAg) SDG is set to a reduction of $90 \%$. $\mathrm{HBeAg}+$ prevalence target is set to 1/1000 individuals. Mean results are obtained from 50 stochastic simulations per intervention combination (vaccination, PMTCT) with parameters sampled from the posteriors shown in Additional file 2: Figure S1. Start of interventions in the stochastic simulations is in year 1995 to simulate an appropriate time scale to address impact by 2030

Projecting the probability of achieving elimination targets based on combinations of neonatal vaccination, PMTCT and enhanced vaccination

We simulated the impact of combining neonatal vaccination and PMTCT with additional vaccine deployment (Fig. 6A1, A2), through the routine vaccination of older children ( $\geq 6$ years of age), and one-off catch-up vaccination of children ( $<6$ years) and others (>6 years). Adding catch-up vaccination campaigns makes no impact on the probability of reaching SDGs (Fig. 6A1, A2, blue and cyan lines). Routine vaccination at 6 years of age, even when delivered at $100 \%$ coverage, is markedly less effective than any other projected intervention (Fig. 6A1, A2, magenta line).

\section{Projecting the impact of HIV on the probability of achieving elimination targets}

A baseline scenario was defined by the epidemiological setting fitted by our model in the context of Kimberley, using local HIV prevalence for each of the modelled age groups (Fig. 6B1, B2, solid line). In a sensitivity exercise, alternative scenarios were considered in which baseline HIV prevalence was altered to zero or higher prevalence. When compared with no HIV (Fig. 6B1, B2, dotted line), the presence of HIV at the prevalence seen in Kimberley (Fig. 6B1, B2, solid line) adds an estimated 4 years to achieve a $50 \%$ chance of reaching the goals (Fig. 6B1). A higher baseline HIV prevalence $(\times 2, \times 3$ and $\times 4$ baseline data for Kimberley) was used to investigate the potential impact of coinfection in high-risk populations. Increasing HIV prevalence has a negative impact on the success of interventions for $\mathrm{HBV}$, but the effects are relatively modest. In particular, doubling HIV prevalence would shift the $50 \%$ probability endpoint into the future by $\sim 4$ years for the HBsAg incidence target and $\sim 7$ years for the HBeAg prevalence target.

\section{Discussion}

This is a unique study in which we capitalise on detailed clinical cohort data collected in South Africa in order to form a robust view of the nature of vaccine-mediated 

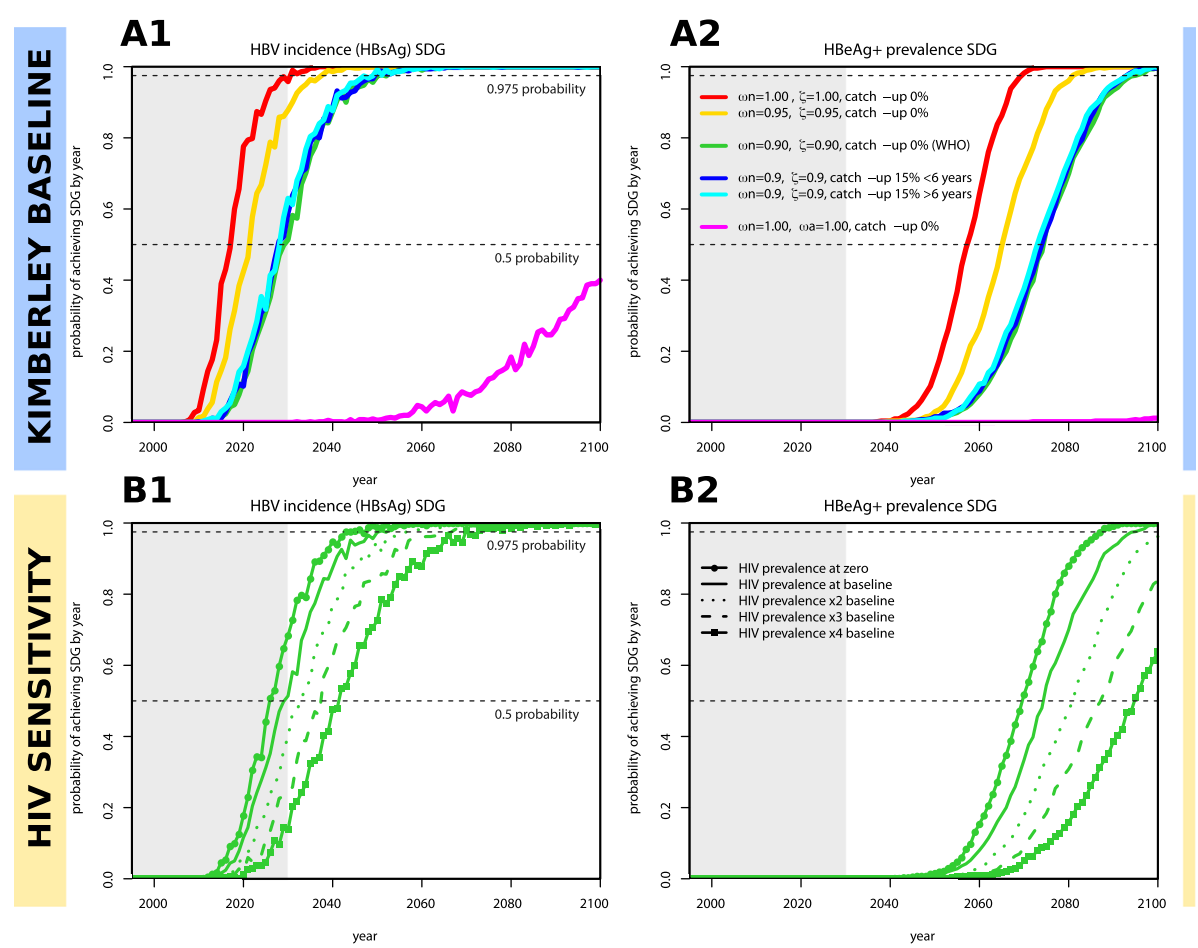

Fig. 6 Yearly estimated probabilities of achieving Sustainable Development Goals (SDGs) for HBV incidence (HBsAg) and HBeAg+ prevalence targets based on particular combinations of interventions and local HIV prevalence levels. A total of 1000 stochastic simulations are run independently for each set of particular interventions (coloured legend, subplot A2), with each using a random parameter sample from the posteriors shown in Additional file 2: Figure S1. Interventions start in year 1995. For every year post-intervention start, the proportion of simulations that have achieved the SDGs is recorded and taken to be the probability. A1 Probability of reaching HBV incidence (HBsAg) SDG in time (goal is set to a reduction of $90 \%$ ). A2 Probability of reaching HBeAg+ prevalence target in time (goal is set to 1/1000 individuals). B1, B2 Same as subplots A1, A2 but addressing sensitivity to HIV prevalence levels in the population for a particular intervention (green, $\omega n=0.9, \zeta=0.9$, catch-up $0 \%(\mathrm{WHO})$ ). Solid line is the same as in subplots A1, A2 (named HIV prevalence at baseline). Other lines present results assuming zero HIV prevalence (full line with points) or higher prevalences (dotted, dashed, line with squares). On all four panels, the dashed horizontal lines mark 0.5 and 0.975 probability of achieving SDGs and the grey shaded area marks the time period before 2030. In the interventions, $\omega n$ is routine vaccination of neonates, $\zeta$ the PMTCT effort, $\omega a$ routine vaccination of +6 years of age, and catch-up a one-off event of vaccination in some age groups or general population

immunity, and develop a mathematical model of HBV transmission and prevention. Overall, we demonstrate that the optimum population intervention is high coverage neonatal vaccination and that this can be strengthened by robust deployment of PMTCT. However, we project long time-scales to achieve elimination targets, congruent with the large established reservoir of chronic HBV infection, lack of curative therapy, infection that can persist for the entire life-span of the host, and interventions that target only a small proportion of the population. Although a high coverage of neonatal vaccination combined with robust PMCTC shows potential promise, the projected timeframe for elimination is currently substantially beyond the 2030 milestone. Complete extinction of infection is far beyond reach based on currently available interventions, and current efforts should be focused on control of HBV as a public health issue rather than complete elimination.

By assimilating the results of a clinical cohort study and a model, we develop a more complete picture than either individual approach would provide in isolation. Only by viewing the two conclusions together can we correctly infer that vaccination is of profound importance in protecting individual children and significantly reducing the burden of infection in paediatric cohorts, but also that continuing to pursue this strategy alone is not sufficient to bring about HBV elimination, or even robust control, within the desired time-scale.

Compared to published models of other vaccinepreventable diseases [23], there is a marked deficit in the existing evidence for $\mathrm{HBV}$, with few other modelling efforts represented in the literature [24, 25]. Reassuringly, our findings are consistent with those of another recent simulation of HBV prevention [19]; we concur in concluding that current vaccine-based interventions will result in a modest reduction in HBV prevalence by the year 2030. However, there are also some important differences that distinguish our work from previous efforts: 
i. Our evaluation provides the advantages of both clinical data and a mathematical model, with close links between our cohort and simulations, and strengths in interpretation of data derived through different approaches. In so doing, we have also been able to specifically address the impact of co-endemic HIV.

ii. We focus on a particular population for which we derive unknown epidemiological parameters and apply a robust data-driven approach to others. Our Bayesian framework therefore stands alone (as a tool) that can be applied to any population for which empirical support of key HBV epidemiological parameters is missing. By supplying the model's code, we can facilitate the use of the tool by others.

iii. As outputs, we have used targets for reductions in both HBsAg incidence and HBeAg-positive prevalence and have projected the impact of interventions based specifically on the WHO proposal for $90 \%$ vaccination of neonates and $90 \%$ PMTCT coverage by 2030. Previous studies [18, 19] have focused instead on ad hoc control thresholds or impact on the public health problem through reduction of HBV-related deaths. Our results thus contribute to an ongoing discussion regarding which goals should be set, and their underlying public health implications. The model suggests that reaching either of the elimination targets will require different intervention coverage and different time scales. In particular, the target for reducing HBsAg incidence is easier to achieve than reducing HBeAg prevalence.

iv. This is an important parsimonious, data-driven tool, offering the potential to scrutinise different strategies independently from one another.

\section{Population epidemiology of HBV}

Variation in prevalence of HBV has been reported from different regions of South Africa, ranging from 2.0-35.2\%, depending upon the population sampled [26, 27]. The adult HBsAg prevalence of $8-11 \%$ that we have recorded in our own previous studies of the region $[6,28]$ is represented in this current analysis; while recognising that there is considerable heterogeneity between populations, we believe this epidemiology also broadly reflects other sub-Saharan African settings, where an adult prevalence of $\geq 8 \%$ would be typical. The much lower prevalence of infection in children demonstrates the success of inclusion of HBV vaccine in the EPI schedule [10, 21, 29].

\section{Impact of HIV on population interventions for HBV}

Our results support previous evidence that HBV vaccinemediated immunity wanes over time independently of
HIV serostatus, but faster for HIV-positive individuals [30]. Impaired vaccine responses have previously been reported in HIV-positive individuals [16, 26, 31-33], but it is also possible that vaccine coverage is lower in HIV-infected children [34]. However, waning of anti-HBs titres does not necessarily correlate with loss of clinical protection; anamnestic responses are thought to occur in a proportion of those vaccinated [35], although this memory may be attenuated by HIV [36, 37].

ART has previously been associated with improved HBV vaccine responses [38, 39], although we did not replicate this finding in our cohort. This can potentially be explained by a previous study in the same setting, demonstrating that immune reconstitution takes a median of 5 years after ART initiation [40]. Our current study is underpowered to detect any ART effect, given both the relatively short durations of therapy and the small number of untreated children. Interestingly, despite the lack of direct association with ART, children with lower HIV viral loads had significantly higher anti-HBs titres, in keeping with previous studies [15, 38].

Our cohort highlights day-to-day challenges of drug provision and monitoring in this setting: we did not have access to detailed prospective ART treatment data, guidelines have changed numerous times since 2002, and lamivudine (3TC) was intermittently used as a substitute for nevirapine (NVP) due to supply issues. During the period covered by our study, ART was only introduced in children achieving certain immunological criteria, while new guidelines recommend that all HIV-infected children are started on ART [41]. The immune reconstitution of this population over time is likely to reduce differences between HIV-positive and HIV-negative groups. ART treatment is relevant to outcomes in individuals with HIV/HBV coinfection, as first line ART regimens include either 3TC or tenofovir (TDF), both of which have activity against HBV. Alternative approaches for HBV prevention in HIV-positive subjects, such as supplementing the current schedule with booster vaccinations and increased vaccine doses, have been trialled with variable results $[15,16]$.

Our projections propose that HIV does have a negative effect on HBV interventions, although HIV prevalence only marginally increases time to reach elimination targets, which may not be significant in light of the long overall time-frames projected, even in the absence of HIV. The high HIV prevalences modelled can occur in specific high-risk groups including sex workers and men who have sex with men [42], and it is likely that increased intervention will be required in these groups to minimise HBV transmission. 


\section{Changes required to meet international goals}

The model suggests long time-lines, enumerated in centuries rather than decades, before control targets are reached using vaccination or PMTCT alone. Combinations of these interventions show much shorter time scales. Based on currently available interventions, major scaling up of both neonatal vaccination and PMTCT efforts will be required. Importantly, the prevalence of $\mathrm{HBeAg}$-positive carriers, who are at an elevated risk of chronic liver disease and hepatocellular carcinoma, as well as being at higher risk of transmitting their infection, will decline at a slower rate. Setting a control target based on reduction in the number of new HBV cases (i.e. HBsAg incidence) can therefore lead to the most optimistic projections but distract attention from the importance of reducing HBeAg prevalence.

Our results also underscore that a major public health impact is possible, even without achieving elimination. Careful adjusting of expectations and aims, according to the scale on which particular changes occur, may inform the setting of realistic targets (e.g. reduction in the prevalence of $\mathrm{HBeAg}$ could be the most informative outcome measure). The wrong choice of either target or timescale may result in unnecessary abandonment of a strategy that could have a major impact in a few decades. In addition to informing rational use of interventions that have a positive population impact, our study is also important in cautioning against the use of strategies that may have little or no lasting population impact. This is illustrated by our results for catch-up HBV vaccination, which adds little in situations where high coverage of both neonatal immunisation and PMTCT can be attained.

\section{Caveats and limitations}

Different approaches to recruitment of our HIVpositive and HIV-negative cohorts may have introduced unintentional bias. The $\mathrm{KReC}$ children may be less healthy than HIV-negative children in the community, and this approach to recruitment predominantly selected younger children (on average 9.4 months younger than the HIV-positive cohort). We set out to focus on children aged $<60$ months in order to collect data from the RTHB. However, in practice, we did not capture good RTHB data and data collection from the RTHB is itself subject to bias, as families who attend with such records may be those who are most likely to have immunised their children. Numerous complex social factors are also relevant in determining immunisation status; babies born to mothers who have HIV and/or HBV are more likely to be in disadvantaged by poverty, and by illness and death in the family, such that they might be less likely to present for (or respond to) vaccination. However, in this setting (and others where antenatal HBV screening is not routinely deployed $[8,43,44])$, we deem it unlikely that there is a significant difference in vaccination rates between infants born to HBV-positive versus HBV-negative mothers.

HBV DNA is a more sensitive screening tool than HBsAg but was not practical due to high cost and lack of availability in this setting. The relatively small numbers in each age group and the lack of longitudinal follow-up for individual children puts limitations on the data showing anti-HBs waning over time, but the trends we observe here are biologically plausible and consistent with existing literature [30, 45].

Although we have estimated and parameterised the impact of HIV status on HBV vaccine-induced protection, we have not modelled other factors related to HIV infection. Namely, we have not included the potential for increased susceptibility to HBV infection or increased risk of vertical transmission. These factors would have required further model classes and specific parameterisation, for which little literature support exists. We have also not considered the influence of population migration on the success of $\mathrm{HBV}$ interventions to reach the elimination targets. Migration of non-immune and/or infected individuals into an area would delay the time to achieve the targets estimated by our modelling approach.

We make no age-specific assumptions about force of transmission. Given that reliable data do not exist to inform this parameterisation, we could potentially add a lot of additional uncertainty by trying to estimate age-specific variables (by need of additional age-dependent model parameters). Keeping parameterisation simple was an intended approach, to avoid introducing uncertainty and to make the model applicable as a tool in other settings. We therefore elected to apply and estimate an agehomogeneous force of transmission. Consequently, the model assumes a uniform risk of HBV infection and transmission potential in the $>6$ years age group. In reality, particular sub-groups in this category are at increased risk of HBV infection, including sex workers, people who inject drugs and healthcare workers [46]. It is likely that these minority risk groups contribute little to the overall burden of HBV infection and transmission in most settings, but further analysis would be required to quantify the specific impact of targeting these groups for intervention. While our modelled projections suggest that generalised catch-up vaccination of adults is insufficient to eliminate HBV at the population level, targeted vaccination of vulnerable groups remains important to protect individuals, and may contribute to public health efforts.

We used 50 stochastic simulations to model the mean and variation of interventions. Although this is a relatively small number, we found no significant differences 
between the resulting mean and variation for each new simulation after a total of approximately 30 . This was likely a result of the stable posteriors obtained, and then sampled to run these simulations (Additional file 2: Figure S1). On these grounds, it is unlikely that extending the number of simulations would alter the final results.

\section{Conclusions}

Our results affirm the success of the HBV vaccine programme in reducing the prevalence of HBV in children, with current paediatric prevalence rates of $<1 \%$. However, we also highlight that cases of HBV transmission persist and that a proportion of children are potentially at risk of infection as a result of low anti-HBs titres, either as a result of missing or incomplete immunisation, or because of poor antibody titres following vaccination. We predict that current elimination targets, in particular when framed around reductions of HBeAg-positive prevalence, are unlikely to be achieved by 2030 . For optimum impact, we suggest that elimination targets could also incorporate $\mathrm{HBeAg-positive} \mathrm{car-}$ riers, which are a major proxy for the public health burden of HBV. Our study highlights the essential need to collect better data that can help to inform progress towards targets, to optimise deployment of vaccination and PMTCT, and to invest substantially in education, case finding and treatment. The prospects of control would be substantially enhanced by improvements in therapy, and ultimately the only route to elimination of HBV may be to develop a cure.

\section{Additional files}

Additional file 1: Clinical cohort metadata. Metadata for three paediatric cohorts recruited in Kimberley, South Africa, including longitudinal CD4+ T cell and viral load data for paediatric HIV cohort age $\leq 60$ months in Kimberley, South Africa. HBV vaccine responses.CSV. HBV vaccine responses.xlsx. COSAC cohort Tables 1 and 2.docx. These files are available on-line using the following link: https://doi.org/10.6084/m9.figshare.5601679. (ZIP 239 kb)

Additional file 2: Full model description and additional supplementary Figure S1-S6. This document includes a complete model description, including parameters, assumptions, mathematical expressions and fitting details, including further results and figures supporting the main text: Figure S1. Posteriors from the Bayesian Markov Chain Monte Carlo fitting to the Kimberley cohort data. Figure S2. Fitting HBV vaccine response according to HIV serostatus. Figure S3. Sensitivity of interventions with deterministic output. Figure S4. Post-intervention stochastic impact on HBV prevalence ( $\mathrm{HBsAg})$, with time to reach sustainable development goals when using routine neonatal vaccination and PMTCT independently. Figure S5. Sensitivity of mean intervention impact on HBV incidence (HBsAg) and $\mathrm{HBeAg}+$ prevalence, with estimated mean year to reach sustainable development goals for combinations of routine +6 years vaccination and PMTCT. Figure S6. Sensitivity of mean intervention impact on $\mathrm{HBV}$ incidence $(\mathrm{HBs} \mathrm{Ag})$ and $\mathrm{HBeAg}+$ prevalence, with estimated mean year to reach sustainable development goals for combinations of routine neonatal vaccination and PMTCT plus a complete catch-up campaign. (PDF 3606 kb)

\section{Abbreviations}

3TC: Lamivudine; Anti-HBC: Antibody to hepatitis B core antigen (antibody mediated by exposure to infection); Anti-HBs: Antibody to hepatitis B surface antigen (vaccine-mediated antibody); ART: Anti-retroviral therapy;

COSAC: Coinfection in South African children; EPI: Expanded Programme on Immunisation; GHSSVH: Global Health Sector Strategy on Viral Hepatitis; HBeAg: Hepatitis B envelope antigen; HBsAg: Hepatitis B surface antigen; HBV: Hepatitis B virus; HIV: Human Immunodeficiency Virus (type 1); KReC: Kimberley Respiratory Cohort; ODE: Ordinary differential equation; OUH: Oxford University Hospitals; PMTCT: Prevention of mother to child transmission; RTHB: Road to Health Book; SDGs: Sustainable Development Goals; WHO: World Health Organization

\section{Acknowledgements}

Not applicable.

\section{Funding}

PCM, PK and PJRG are funded by the Wellcome Trust (grant numbers 110110/Z/15/Z to PM, 109965MA to PK, and 104748MA to PJRG); https://wellcome.ac.uk. Recruitment and serological testing of the KReC cohort was covered by a project grant awarded to PCM from the Rosetrees Trust http://www.rosetreestrust.co.uk/. SG and JL received funding from the European Research Council under the European Union's Seventh Framework Programme (FP7/2007-2013)/ERC grant agreement no. 268904-DIVERSITY https://erc.europa.eu/. PK is also funded by an NIHR Senior Fellowship https://www.nihr.ac.uk/. The funders had no role in study design, data collection and analysis, decision to publish, or preparation of the manuscript.

\section{Availability of data and materials}

The datasets generated and/or analysed during the current study are available in the Figshare repository: https://doi.org/10.6084/m9.figshare.5601679.

Our code is available at https://sourceforge.net/u/lourencoj/profile/ entitled 'HBV interventions model'.

\section{Authors' contributions}

PM, SG, and PJ conceived the idea. PM, PG, and PJ applied for ethical permission. $P M, P G, P J$, and PK secured funding. AM, JL, and PM wrote the manuscript draft. $\mathrm{LH}, \mathrm{EA}, \mathrm{SD}, \mathrm{AvZ}$, and PJ recruited children and collected the clinical data. EA, CA, and $A M$ analysed the clinical data. SW and $\mathrm{KJ}$ undertook laboratory assays for HBV serology. JL and SG with input from AM, PM, and MAA developed and tested mathematical model. All authors read and approved the final manuscript.

Ethics approval and consent to participate

Ethics approval was obtained from the Ethics Committee of the Faculty of Health Science, University of the Free State, Bloemfontein, South Africa (HIV Study Ref: ETOVS Nr 08/09 and COSAC Study Ref: ECUFS NR 80/2014), and from the Oxfordshire Research Ethics Committee A, ref 06/Q1604/12. Written consent for enrollment into the study was obtained from the child's parent/guardian.

\section{Consent for publication}

Not applicable.

\section{Competing interests}

PCM is an Associate Editor for BMC Infectious Diseases and undertakes consultancy work for Immunocore.

\section{Publisher's Note}

Springer Nature remains neutral with regard to jurisdictional claims in published maps and institutional affiliations.

\section{Author details}

${ }^{1}$ Nuffield Department of Medicine, Peter Medawar Building for Pathogen Research, South Parks Road, Oxford OX1 3SY, UK. ²Department of Zoology, Peter Medawar Building for Pathogen Research, South Parks Road, Oxford OX1 3SY, UK. ${ }^{3}$ Department of Paediatrics, Kimberley Hospital, Kimberley 8300, South Africa. ${ }^{4}$ Department of Paediatrics, Peter Medawar Building for Pathogen Research, South Parks Road, Oxford OX1 3SY, UK. ${ }^{5}$ Global Healthcare Public Foundation, Makindu Lane, Kololo, Kampala, Uganda. ${ }^{6}$ Department of Infectious Diseases and Microbiology, Oxford University Hospitals NHS Foundation Trust, John Radcliffe Hospital, Headley Way, Oxford OX3 9DU, UK. 


\section{Received: 24 September 2018 Accepted: 22 January 2019}

\section{Published online: 21 February 2019}

\section{References}

1. World Health Organization. Combating hepatitis B and C to reach elimination by 2030: Advocacy Brief 2016.

2. World Health Organization. Global health sector strategy on viral hepatitis 2016-2021. 2016;https:/www.who.int/hepatitis/strategy2016-2021/en/.

3. Ott JJJ, Horn J, Krause G, Mikolajczyk RTT. Time trends of chronic HBV infection over prior decades - a global analysis. J Hepatol. 2017;66(April 2016):48-54.

4. World Health Organization. Introduction of hepatitis B vaccine into childhood immunization services Management guidelines, including information for health workers and parents. Department of Vaccines and Biologicals, https://apps.who.int/iris/handle/10665/66957. 2001.

5. Schweitzer A, Horn J, Mikolajczyk RT, Krause G, Ott JJ. Estimations of worldwide prevalence of chronic hepatitis B virus infection: a systematic review of data published between 1965 and 2013. Lancet. 2015;386:1546-55.

6. Matthews PC, Beloukas A, Malik A, Carlson JM, Jooste P, Ogwu A, et al. Prevalence and characteristics of hepatitis $B$ virus (HBV) coinfection among HIV-positive women in South Africa and Botswana. PLoS One. 2015;10(7): e0134037.

7. Statistics South Africa. Mid-year population estimates. 2016; https://www. statssa.gov.za/.

8. O'Hara GA, McNaughton AL, Maponga T, Jooste P, Ocama P, Chilengi R, et al. Hepatitis B virus as a neglected tropical disease. PLOS Negl Trop Dis. 2017;11(10):e0005842 https://doi.org/10.1371/journal.pntd.0005842.

9. Mokaya J, McNaughton AL, Burbridge L, Maponga T, O'Hara G, Andersson $\mathrm{M}$, et al. A blind spot? Confronting the stigma of hepatitis B virus (HBV) infection - a systematic review. Wellcome Open Res. 2018;3(0):29.

10. Burnett RJ, Kramvis A, Dochez C, Meheus A. An update after 16 years of hepatitis B vaccination in South Africa. Vaccine. 2012;30(Supplement 3):C45-51.

11. Chotun N, Nel E, Cotton MF, Preiser W, Andersson MI. Hepatitis B virus infection in HIV-exposed infants in the Western Cape, South Africa. Vaccine. 2015;33(36):4618-22.

12. Public Health England. Hepatitis B. In: Green Book: Immunisation against infectious disease; 2016. p. 161-85.

13. Jack A, Hall A, Maine $N$, Mendy $M$, Whittle $H$. What level of hepatitis $B$ antibody is protective? J Infect Dis. 1999;179:489-92.

14. World Health Organization. Hepatitis B vaccines: WHO position paper, July 2017 - Recommendations. Vaccine. 2019;37(2):223-5. https://doi.org/10. 1016/j.vaccine.2017.07.046

15. Catherine F-X, Piroth L. Hepatitis B virus vaccination in HIV-infected people: a review. Hum Vaccin Immunother. 2017. https://doi.org/10.1080/21645515. 2016.1277844

16. Lao-araya M, Puthanakit T, Aurpibul L, Taecharoenkul S, Sirisanthana T, Sirisanthana $V$. Prevalence of protective level of hepatitis $B$ antibody 3 years after revaccination in HIV-infected children on antiretroviral therapy. Vaccine. 2011;29(23):3977-81.

17. Lee C, Gong Y, Brok J, Boxall EH, Gluud C, Eh B, et al. Hepatitis B immunisation for newborn infants of hepatitis B surface antigen-positive mothers (Review). Cochrane Database Syst Rev. 2006;(2). https://doi.org/10. 1002/14651858.CD004790.pub2.

18. Polaris T, Collaborators O. Global prevalence, treatment, and prevention of hepatitis B virus infection in 2016: a modelling study. Lancet Gastroenterol Hepatol. 2018;(18)30056-6 https://doi.org/10.1016/S2468-1253.

19. Nayagam S, Thursz M, Sicuri E, Conteh L, Wiktor S, Low-Beer D, et al. Requirements for global elimination of hepatitis B: a modelling study. Lancet Infect Dis. 2016;16(12):1399-408.

20. Sharp CP, Gregory WF, Hattingh L, Malik A, Adland E, Daniels S, et al. PARV4 prevalence, phylogeny, immunology and coinfection with HIV, HBV and HCV in a multicentre African cohort. Wellcome Open Res. 2017;2(0):26.

21. Jooste P, van Zyl A, Adland E, Daniels S, Hattingh L, Brits A, et al. Screening, characterisation and prevention of hepatitis B virus (HBV) co-infection in HIV-positive children in South Africa. J Clin Virol. 2016;85:71-4.

22. Hadler SC, Francis DP, Maynard JE, Thompson SE, Judson FN, Echenberg dean $F$, et al. Long-term immunogenicity and efficacy of hepatitis B vaccine in homosexual men. N Engl J Med 1986;315(4):209-214.

23. Hashim A, Dang V, Bolotin S, Crowcroft NS. How and why researchers use the number needed to vaccinate to inform decision making-a systematic review. Vaccine. 2015;33(6):753-8.
24. Nayagam $S$, Conteh $L$, Sicuri E, Shimakawa $Y$, Suso P, Tamba $S$, et al. Cost-effectiveness of community-based screening and treatment for chronic hepatitis B in the Gambia: an economic modelling analysis. Lancet Glob Health. 2016;4(8):e568-78.

25. Anderson S, Harper LM, Dionne-Odom J, Halle-Ekane G, Tita ATN. A decision analytic model for prevention of hepatitis B virus infection in Sub-Saharan Africa using birth-dose vaccination. Int J Gynecol Obstetrics. 2018;141(1): 126-32.

26. Mayaphi SH, Rossouw TM, Masemola DP, Olorunju SA, Mphahlele MJ, Martin DJ. HBV/HIV co-infection : the dynamics of HBV in South African patients with AIDS. S Afr Med J. 2012;102(3):157-62.

27. Mphahlele MJ, Lukhwareni A, Burnett RJ, Moropeng LM, Ngobeni JM. High risk of occult hepatitis B virus infection in HIV-positive patients from South Africa. J Clin Virol. 2006;35(1):14-20.

28. Matthews PC, Geretti AM, Goulder PJR, Klenerman P. Epidemiology and impact of HIV coinfection with hepatitis B and hepatitis $C$ viruses in subSaharan Africa. J Clin Virol. 2014;61:20-33.

29. Amponsah-Dacosta E1, Lebelo RL, Rakgole JN, Burnett RJ, Selabe SG, Mphahlele MJ. Evidence for a change in the epidemiology of hepatitis B virus infection after nearly two decades of universal hepatitis B vaccination in South Africa. J Med Virol. 2014;86:918-24. https://doi.org/10.1002/jmv. 23910

30. Chaouch H, Hachfi W, Fodha I, Kallala O, Saadi S, Bousaadia A, et al. Impact and long-term protection of hepatitis B vaccination: 17 years after universal hepatitis B vaccination in Tunisia. Epidemiol Infect. 2016;144(16):3365-75.

31. Sokal EM, Paganelli M, Wirth S, Socha P, Vajro P, Lacaille F, et al. Management of chronic hepatitis B in childhood: ESPGHAN clinical practice guidelines: consensus of an expert panel on behalf of the European Society of Pediatric Gastroenterology, Hepatology and Nutrition. J Hepatol. 2013; 59(4):814-29.

32. Büchner A, Omar FE, Vermeulen J, Reynders DT. Investigating hepatitis B immunity in patients presenting to a Paediatric Haematology and oncology unit in South Africa. S Afr Med J. 2014;104(9):628-31.

33. Beghin J-C, Ruelle J, Sokal E, Bachy A, Krishna M, Hall L, et al. Effectiveness of the South African expanded program of immunization against hepatitis $B$ in children infected with human immunodeficiency virus-1 living in a resource-limited setting of Kwazulu-Natal. J Med Virol. 2017;89:182-5.

34. Ndirangu J, Barnighausen T, Tanser F, Tint K, Newell ML, Bärnighausen T, et al. Levels of childhood vaccination coverage and the impact of maternal HIV status on child vaccination status in rural KwaZulu-Natal, South Africa. Trop Med Int Health. 2009;14(11):1383-93.

35. Banatvala J, Van Damme P, Oehen S. Lifelong protection against hepatitis B: the role of vaccine immunogenicity in immune memory. Vaccine. 2000;19(7-8):877-85.

36. Lao-araya M, Puthanakit T, Aurpibul L, Sirisanthana T, Sirisanthana V. Antibody response to hepatitis $B$ re-vaccination in HIV-infected children with immune recovery on highly active antiretroviral therapy. Vaccine. 2007; 25(29):5324-9.

37. Abzug MJ, Warshaw MG, Rosenblatt HM, Levin MJ, Nachman S, Pelton SI, et al. Immunogenicity and immunologic memory after hepatitis $B$ virus booster vaccination in HIV-infected children receiving highly active antiretroviral therapy. J Infect Dis. 2009;200(6):935-46.

38. Kim HN, Harrington RD, Van Rompaey SE, Kitahata MM. Independent clinical predictors of impaired response to hepatitis B vaccination in HIV-infected persons. Int J STD AIDS. 2008;19(9):600-4.

39. Pippi F, Bracciale L, Stolzuoli L, Giaccherini R, Montomoli E, Gentile C, et al. Serological response to hepatitis B virus vaccine in HIV-infected children in Tanzania. HIV Med. 2008;9(7):519-25.

40. Mori M, Adland E, Paioni P, Swordy A, Mori L, Laker L, et al. Sex differences in antiretroviral therapy initiation in pediatric HIV infection. PLoS One. 2015; 10(7):e0131591.

41. World Health Organization. Consolidated Guidelines on the Use of Antiretroviral Drugs for Treating and Preventing HIV Infection, Recommendations for a Public Health Approach. 2nd edition. Geneva: World Health Organization; 2016. ISBN-13: 978-92-4-154968-4.

42. South African National AIDS Council. Let our actions count: reflections on NSP 2012-2016 and moving forward to NSP 2017-2022. 2016.

43. Chotun N, Preiser W, van Rensburg CJ, Fernandez P, Theron GB, Glebe D, et al. Point-of-care screening for hepatitis $B$ virus infection in pregnant women at an antenatal clinic : a South African experience. PLoS One. 2017;12(7): e0181267 https://doi.org/10.1371/journal. pone.0181267 Editor. 
44. Diale Q, Pattinson R, Chokoe R, Masenyetse L, Mayaphi S. Antenatal screening for hepatitis B virus in HIV-infected and uninfected pregnant women in the Tshwane district of South Africa. S Afr Med J. 2015;106(1):97-100.

45. Katoonizadeh A, Sharafkhah M, Ostovaneh MR, Norouzi A, Khoshbakht N, Mohamadkhani A, et al. Immune responses to hepatitis B immunization 10-18 years after primary vaccination: a population-based cohort study. J Viral Hepat. 2016;23:805-11.

46. World Health Organization. Hepatitis B factsheet. 2017;http://www.who.int/ mediacentre/factsheets/fs204/en

Ready to submit your research? Choose BMC and benefit from:

- fast, convenient online submission

- thorough peer review by experienced researchers in your field

- rapid publication on acceptance

- support for research data, including large and complex data types

- gold Open Access which fosters wider collaboration and increased citations

- maximum visibility for your research: over $100 \mathrm{M}$ website views per year

At $\mathrm{BMC}$, research is always in progress.

Learn more biomedcentral.com/submissions 